\title{
Ann-Christin Leßmann
}

\section{Diskursive Anforderungen in der Grundschule}

\begin{abstract}
Unterrichtskommunikation unterscheidet sich in vielen Aspekten von der Alltagskommunikation. Diese kontextspezifischen Anforderungen werden Schülerinnen und Schülern mit Ausnahme organisatorischer Regeln selten explizit vermittelt; sie sind vielmehr Teil der schulischen Sozialisation. Das gilt gerade für diskursive Anforderungen, wenn also von Schülerinnen und Schülern eine Beteiligung an kommunikativen Gattungen wie Erzählen, Erklären, Argumentieren erwartet wird. Für die erfolgreiche Partizipation am Unterricht ist es jedoch für die Schülerinnen und Schüler elementar, die sequenziellen Mechanismen im Unterrichtsgespräch zu erkennen und ihren Part angemessen auszuführen. Auf Grundlage eines Korpus von 18 videographierten Unterrichtsstunden zweier Grundschulklassen im Verlauf des ersten Schuljahres beleuchtet der Beitrag, welche diskursiven Anforderungen Lehrpersonen im Unterrichtsgespräch an die Schülerinnen und Schüler stellen. Dabei soll vor allem der Grad der Explizitheit bzw. Implizitheit globaler Zugzwänge untersucht werden: Vielfach erscheinen die Anforderungen nur implizit, so dass sie oftmals erst in den lehrerseitigen Rückmeldungen gegenüber einzelnen Schüleräußerungen sichtbar werden. Hierzu zeigt der Beitrag einige exemplarische Verfahren und beleuchtet damit ganz im Sinne der sequenziellen Rekonstruktion den Zusammenhang zwischen lehrerseitigem Zugzwang, Schüleräußerung und Lehrerrückmeldung. Es wird auch gezeigt, welche Schwierigkeiten sich für einige Schülerinnen und Schüler durch eine zu implizite Etablierung diskursiver unterrichtlicher Anforderungen ergeben.
\end{abstract}

Keywords: Unterrichtskommunikation, diskursive Anforderungen, Diskurseinheiten, bildungssprachliche Praktiken, Lehrerrückmeldungen, Implizitheit, Explizitheit

\section{Einleitung}

Diskursiven Praktiken (vgl. zu diesem und anderen grundlegenden Konzepten das Glossar in diesem Band) wie Berichten, Erklären und Argumentieren wird

\footnotetext{
Ann-Christin Leßmann, Universität Bielefeld, Universitätsstraße 25, 33615 Bielefeld, ann-christin.lessmann@uni-bielefeld.de

Ә Open Access. (C) 2021 Ann-Christin Leßmann, publiziert von De Gruyter. (cc) BY-NC-ND Dieses Werk ist lizensiert unter einer Creative Commons Namensnennung - Nicht-kommerziell - Keine Bearbeitung 4.0 International Lizenz.

https://doi.org/10.1515/9783110707168-010
} 
ein hoher Stellenwert für das Lernen eingeräumt. Mit diskursiven Praktiken sind „Verfahren angesprochen, bei denen äußerungsübergreifende Einheiten, sog. Diskurseinheiten, interaktiv hervorgebracht werden“ (Heller et al. 2017: 140). Der Begriff ,Praktiken“ verweist darauf, dass es sich um gesellschaftlich entwickelte ,Lösungsmuster‘ zur Bewältigung kommunikativer Probleme handelt (vgl. Deppermann, Feilke \& Linke 2016).

Diskursive Praktiken sind anteilig auch in den Bildungsstandards verankert (KMK 2004). Allerdings ist unklar, inwiefern sie im Klassengespräch gefördert werden. Heller et al. (2017) weisen z. B. darauf hin, dass Lehrkräfte den Umgang mit diskursiven Praktiken auf einer globalstrukturellen Ebene kaum in den Blick nehmen und stattdessen stark auf inhaltliche Aspekte fokussieren. Der Beitrag versucht anhand exemplarischer Analysen diesen impliziten, lehrerseitigen Umgang mit diskursiven Praktiken zu beleuchten. Er thematisiert dabei auch die daraus folgenden Schwierigkeiten für Schülerinnen und Schüler, für die gerade im Anfangsunterricht noch nicht vorausgesetzt werden kann, dass sie sicher globale und lokale Zugzwänge voneinander unterscheiden können, da sie sich noch relativ am Anfang des Diskurserwerbs befinden (Hausendorf \& Quasthoff 2005).

Der Artikel expliziert zunächst, was unter diskursiven Anforderungen zu verstehen ist und welche diskursiven Einheiten im Unterrichtsgespräch der Grundschule zu erwarten sind (Abschnitt 2). In Abschnitt 3 wird das dem Artikel zugrundeliegende Datenkorpus vorgestellt. In Abschnitt 4 wird dann die Etablierung diskursiver Anforderungen im Unterrichtsgespräch anhand exemplarischer Fallbeispiele vorgestellt. Ein anschließendes Fazit fasst die Ergebnisse zusammen und zeigt, dass die Förderung diskursiver Praktiken in der Grundschule ausbaufähig ist. Dazu muss ein Bewusstsein für derartige Praktiken geschaffen werden.

\section{Anforderungen in der Grundschule}

Wenn in diesem Artikel von Anforderungen in der Grundschule gesprochen wird, sind damit nicht primär lehrplanmäßige oder curriculare Anforderungen gemeint. Fokussiert werden die Anforderungen, die Lehrpersonen in ihrer Rolle als Vertreter der Institution Schule im laufenden Unterrichtsgespräch stellen. Während das ,Stellen von Anforderungen‘ zu den Aufgaben der Lehrperson gehört, kommt den Schülerinnen und Schülern in der Regel die ,Erfüllung‘ eben dieser Anforderungen zu. Das wird im folgenden Abschnitt 2.1 näher erläutert.

Im vorliegenden Artikel soll insbesondere der Umgang von Lehrpersonen mit diskursiven Einheiten im Grundschulunterricht beleuchtet werden. Daher stellt 
sich die Frage, welche Diskursgattungen überhaupt in der Grundschule erwartet werden können. Auf diesen Aspekt gehe ich in Abschnitt 2.2 ein.

\subsection{Anforderungen stellen und Anforderungen erfüllen: Aufgaben von Lehrpersonen und Schülerinnen und Schülern}

Unterrichtskommunikation ist eine besondere Kommunikationsform. Die in ihr stattfindenden Interaktionen sind zugeschnitten auf die institutionellen Zwecke, die zum einen die Vermittlung von Wissen, zum anderen die Selektion (Ehlich 2009) betreffen. Das zeigt sich schon in der üblichen Konstellation, in der eine Lehrperson viele Schülerinnen und Schüler unterrichtet. Neben der unterschiedlichen Größe der Parteien verdeutlicht dies auch die Asymmetrie der Rollen und Rechte: Die Lehrperson hat die Aufgabe, Wissen an möglichst viele heranwachsende Gesellschaftsmitglieder gleichzeitig zu vermitteln und die Leistung der Schülerinnen und Schüler zu bewerten. Sie ist daher prinzipiell mit mehr Rechten ausgestattet als die Schülerinnen und Schüler, wobei dieses Verhältnis von den Schülerinnen und Schülern ko-konstruiert und dadurch hergestellt wird (Becker-Mrotzek \& Quasthoff 1998). Die Interaktion zwischen Lehrperson und Schülerschaft ist derart routiniert, dass diese Rollenverteilung nicht jedes Mal neu ausgehandelt werden muss (Heller 2014). Gerade im Anfangsunterricht zeigt sich aber, dass die Rollenverteilung kein Selbstläufer ist, sondern Schülerinnen und Schüler ein institutionsangemessenes Verhalten erst lernen müssen. Einige Regeln werden dabei bewusst als ,Gesprächs- und Verhaltensregeln` eingeführt, auf die bei Verstößen vor allem im Rahmen von Disziplinierungen verwiesen wird (Vogt 1998).

Prinzipiell vollzieht sich aber die schulische Sozialisation ,nebenbei“ (Neuland, Balsliemke \& Baradaranossadat 2009), d. h. in Interaktionen, die eigentlich Anderes zum Gegenstand haben: Die Zugzwänge und Rückmeldungen der Lehrperson geben hier Anhaltspunkte für das, was als angemessen gilt. ${ }^{1}$ Dabei geht es nicht nur um ein bestimmtes Verhalten, wie ,auf dem Platz sitzenbleiben' und ,nicht unerlaubt durch die Klasse laufen“, ,sich melden“

\footnotetext{
1 Angemessenheit wird hier und im gesamten Artikel nicht als extern festgelegter normativer Maßstab angesetzt. Vielmehr wird konsequent aus Teilnehmerperspektive rekonstruiert, was die Beteiligten selbst als angemessen miteinander aushandeln. Das kann durchaus von externen Normvorstellungen abweichen. Für Unterricht gilt aufgrund seiner institutionellen Ausrichtung mit asymmetrischer Rechte- und Wissensverteilung, dass Angemessenheit im Wesentlichen von der Lehrperson bestimmt wird (Buttlar 2017a).
} 
statt selbstgewählt zu sprechen etc. Vielmehr stellt die Lehrperson Anforderungen auf verschiedenen Ebenen. Gefordert wird neben dem angemessenen Verhalten z. B. auch die Mitarbeit an der Etablierung von Wissen, was eine Anforderung auf inhaltlicher und diskursiver Ebene darstellt. Heller (2012) hat herausgearbeitet, dass Schüleräußerungen in Bezug auf vier Ebenen (in-)kongruent sein können. Die bekannteste ist sicherlich die genannte Inhaltliche. Daneben sind auch die sprachlich-formale sowie die gesprächsorganisatorische Ebene gut bekannt (vgl. Hausendorf \& Quasthoff 2005: 310-311). Erstere verweist auf die Verwendung sprachlicher Mittel, während letztere auf die kommunikative Ordnung (Becker-Mrotzek \& Vogt 2009) anspielt. Eine vierte Ebene ist die globalstrukturelle. Sie wird von Lehrpersonen am wenigsten explizit fokussiert, wie Hausendorf \& Quasthoff (2005: 310-311) schlussfolgern und auch Gruppeninterviews in Heller et al. (2017) und Quasthoff \& Heller (in diesem Band) nahelegen. Die globalstrukturelle Ebene betrifft die Erfüllung globaler Zugzwänge, d.h. die Umsetzung diskursiver Einheiten wie z. B. Erklären, Argumentieren, Berichten, Beschreiben, Erzählen. Um diese Ebene soll es in diesem Artikel vornehmlich gehen.

Um die Aufmerksamkeit der Schülerinnen und Schüler zu sichern, wird der Unterricht als asymmetrisches Gespräch strukturiert, d. h., schülerseitige Beteiligung eingefordert (vgl. Becker-Mrotzek \& Vogt 2009). Die Anforderungen werden zunächst durch sogenannte Zugzwänge (Quasthoff \& Kern 2007) wie (Lehrer-) Fragen, Aufgabenstellungen, Anweisungen etc. etabliert; d. h. von den Schülerinnen und Schülern wird erwartet, auf den turn der Lehrperson zu reagieren. Diese lehrerseitigen Zugzwänge sind für das Unterrichtsgespräch zentrale Schnittstellen. Sie können neue thematische Sequenzen eröffnen und strukturieren so das Unterrichtsgespräch oder sie initiieren die Bearbeitung eines Schülerbeitrags. Didaktische Handreichungen legen viel Wert auf diesen ersten Schritt in einer unterrichtlichen Sequenz. Die ,Aufgabenstellungen“ sollen klar formuliert sein und etwaige Missverständnisse vermeiden (z. B. Dubs 2009). Nicht immer werden diese didaktischen Zielsetzungen aber umgesetzt. Sie kollidieren teils mit dem Anspruch, die Schülerinnen und Schüler zu aktivieren, ohne $\mathrm{zu}$ viele Hinweise auf die ,richtige Lösung‘ ${ }^{\star} u$ geben, oder einen möglichst offenen und damit für alle Schülerinnen und Schüler ,passenden“ Impuls zu geben (vgl. Ehlich \& Rehbein 1986). Daraus folgt, dass die Anforderungen der Lehrperson an die Schülerinnen und Schüler nicht immer bereits im Zugzwang klar formuliert sind. Vielfach werden sie erst im Nachhinein, d. h. in der Rückmeldung der Lehrperson auf Schüleräußerungen, deutlich (Buttlar 2017a).

Rückmeldungen sind Bewertungen der Schüleräußerungen. Dieses Bewerten ist eine typische Aufgabe der Lehrperson im Unterrichtsgespräch (Streeck 1979). Es geht dabei im Hinblick auf das Unterrichtsgespräch weniger um ein 
Bewerten im Sinne von Schulnoten zur mündlichen Mitarbeit, vielmehr markiert dieses Bewerten die (Un-)Angemessenheit der schülerseitigen Äußerung(en) in Bezug auf das Klassengespräch. Die Lehrperson zeigt so, welche Äußerungen oder Äußerungsteile relevant für das Unterrichtsgespräch sind und welche nicht.

Prinzipiell sind unterrichtliche Sequenzen also (mindestens) dreischrittig aufgebaut. Dementsprechend werden sie von Mehan (1979a) als sogenannte IRE-Sequenzen beschrieben: Auf eine lehrerseitige initiation folgt im Idealfall ein schülerseitiges reply, dem wiederum folgt eine lehrerseitige evaluation. Gerade im Fall einer nicht adäquaten schülerseitigen Bedienung des Zugzwangs können sich allerdings weitere sequenzielle Schritte anschließen, da der Zugzwang der Lehrperson nicht als bedient markiert wird und so die Sequenz nicht abgeschlossen werden kann (Mehan 1979b; McHoul 1990; Macbeth 2004).

Generell findet eine Würdigung einer Schüleräußerung dann statt, wenn sie in den Unterricht integriert wird, d. h. Teil der Unterrichtsöffentlichkeit wird (Vogt 1998). Das setzt jedoch voraus, dass eine Schülerin bzw. ein Schüler die Anforderungen der Lehrperson zumindest teilweise erfüllt. Daraus lässt sich folgern, dass Schülerinnen und Schüler dann erfolgreich am Unterricht partizipieren können, wenn sie die Anforderungen der Lehrperson erkennen und ausführen. Der kommunikative Erfolg zeigt sich insbesondere in den Rückmeldungen der Lehrperson. Für die Rekonstruktion diskursiver Anforderungen muss also stets der vollständige Kontext berücksichtigt werden, d. h. auch die Schüleräußerung(en) und die Lehrerrückmeldung sowie der Vorlauf zur Sequenz. Es stellt sich dabei die Frage, welche diskursiven Anforderungen in den Zugzwängen und Rückmeldungen auf welche Weise etabliert werden. Dieser Frage soll weiter unten anhand exemplarischer Analysen von Unterrichtssequenzen aus der Grundschule nachgegangen werden. Im Fokus der Untersuchung sollen vor allem Sequenzen stehen, in denen diskursive Anforderungen nur implizit gesetzt werden, da sich hier größere Schwierigkeiten für Schülerinnen und Schüler ergeben.

\subsection{Diskursive Gattungen im Grundschulunterricht}

Aus dem vorigen Abschnitt ging hervor, dass Lehrpersonen im Unterrichtsgespräch Anforderungen an die Schülerinnen und Schüler auf verschiedenen Ebenen stellen. Darunter fällt auch die globalstrukturelle, in die sich diskursive Anforderungen einordnen lassen.

Mit ,diskursiven Anforderungen“ ist gemeint, dass die Zugzwänge der Lehrperson globale Anforderungen etablieren, d. h. es werden größere sprachliche Einhei- 
ten eingefordert, die über die Wort- und Satzebene hinausgehen (Quasthoff 2011), sogenannte Äußerungspakete (Morek, Heller \& Quasthoff 2017). Das ist z. B. „ein Argument, das in einer Diskussion entfaltet wird“ (Quasthoff 2011: 213) und als solches zu einer sogenannten Diskurseinheit (Wald 1978), in diesem Fall zu einer Argumentation, gehört ${ }^{2}$. Diskurseinheiten und damit auch Äußerungspakete sind in der Regel Bestandteil gesellschaftlich etablierter Praktiken. Damit sind interaktive Muster gemeint, die sich für die Bearbeitung wiederkehrender ,Probleme“ in Kommunikationssituationen herausgebildet haben. „In diesem Sinne stellen die Konstruktion, Aushandlung und Vermittlung von Wissen wiederkehrende gesellschaftliche ,Probleme` dar, die sozial geregelte kommunikative Lösungsverfahren verlangen“ (Heller et al. 2017: 139). $\mathrm{Zu}$ diesen Lösungsverfahren zählen Heller et al. nun insbesondere diskursive Praktiken wie Erzählen, Erklären, Beschreiben, Argumentieren oder auch Berichten (Heller et al. 2017), die mit Luckmann (1986) als kommunikative Gattungen bezeichnet werden können.

Diskursive Praktiken sind vielfach Gegenstand gesprächsanalytischer Untersuchungen zur Unterrichtskommunikation. Häufig werden dabei (schülerseitiges) Erklären (z. B. Spreckels 2009; Kotthoff 2009; Becker-Mrotzek \& Vogt 2009: $141 \mathrm{ff}$; Morek 2011, 2012; Morek, Heller \& Quasthoff 2017) und Argumentieren (z. B. Heller 2012; Grundler \& Vogt 2006; Grundler 2011; Morek, Heller \& Quasthoff 2017) im Schulunterricht in den Blick genommen, vielfach in Bezug auf die Sekundarstufe I (z. B. Spiegel 1999, 2006; Harren 2009; Heller \& Morek 2015; Erath et al. 2014; Morek 2016; Prediger et al. 2016; Quasthoff et al. submitted). Für die Grundschule steht die diskursive Praktik des Erzählens hingegen mehr im Fokus (z. B. Knapp 2001; Ohlhus \& Stude 2009; Becker \& Wieler 2013), prominent untersucht auch im Erzählkreis, der typisch für den Grundschulunterricht ist (z. B. Fienemann \& Kügelgen 2006; Becker-Mrotzek 2011; Morek 2013). Die Fülle an Studien weist bereits auf den Stellenwert diskursiver Praktiken im Unterrichtsgespräch hin. Als gesellschaftlich verfestigte interaktive Muster zur Lösung wiederkehrender ,Probleme‘ kommen sie häufig vor,

2 Quasthoff (2011) bezieht sich in ihren Ausführungen ausschließlich auf den Begriff der Diskurseinheiten nach Wald (1978). Mittlerweile wurde der Begriff aber von Morek, Heller \& Quasthoff (2017) weiter differenziert: Während sich der Begriff Diskurseinheit auf die gesamte Sequenz inklusive der Relevantsetzung einer Erklärung o. Ä. bezieht, verweist der Begriff Äußerungspaket in Anlehnung an Sacks‘ big packages (Sacks 1995: 354) auf einzelne übersatzmäßige Äußerungen, die im Rahmen einer global organisierten Diskurseinheit wie z. B. einer Erklärung auftreten. Diese Differenzierung wird im vorliegenden Artikel übernommen (vgl. das Glossar in diesem Band). 
auch und gerade im Unterricht. Dort wird ihnen ein zentraler Stellenwert zum Lernen beigemessen.

So stellen Morek \& Heller (2012) in diesem Zusammenhang die Rolle von übersatzmäßigen Einheiten im Diskurs heraus und fassen sie als bildungssprachliche Praktiken auf. Damit weisen sie darauf hin, dass die unterrichtlichen Anforderungen an mündliche Sprache nicht nur auf sprachstruktureller Ebene in Form von Wohlgeformtheit von Äußerungen und Verwendung von Fachlexik bestehen, sondern auch die Einbettung von Äußerungspaketen in den jeweiligen Kontext umfassen (vgl. Quasthoff \& Morek 2015). Im vorigen Abschnitt wurde bereits darauf hingewiesen, dass Schüleräußerungen aufgrund einer globalstrukturellen Unangemessenheit zurückgewiesen werden können. Insofern erscheint die Beherrschung von diskursiven Praktiken für den Schulerfolg entscheidend, wie auch Heller \& Morek (2015: 2) konstatieren: Die „Beherrschung solcher globaler Diskurspraktiken [. . .] ermöglicht die aktive Partizipation an kommunikativen Prozessen im Schulunterricht, die ihrerseits wiederum sprachliche und fachliche Lernprozesse in Gang setzen.“

Die epistemische Funktion von diskursiven Praktiken beleuchten auch Prediger et al. (2016). Sie zeigen die Relevanz dieser Praktiken für den Fachunterricht, da „Sprache nicht nur produktiv und rezeptiv zu kommunikativen Zwecken gebraucht wird, sondern auch ein Denkmittel in Wissenskonstruktionsprozessen ist“ (ebd.: 286). Diese Wissenskonstruktionsprozesse finden maßgeblich im lehrergeleiteten Fachunterricht statt, indem kognitiv anspruchsvolle fachliche Inhalte mittels diskursiver Praktiken vermittelt und erarbeitet werden (können) (Heller \& Morek 2015; Prediger et al. 2016). Aus diesem (theoretischen) Blickwinkel heraus ist bzw. sollte das lehrerseitige Stellen diskursiver Anforderungen wesentlicher Bestandteil des Unterrichtsgesprächs sein.

Wie aber sieht es in der Praxis aus? Welche diskursiven Anforderungen werden tatsächlich gestellt? Morek (2012: 24) hält fest, dass bisher wenig darüber bekannt sei, „in welchem Rahmen Schülerinnen und Schüler gleich welcher Schulstufe im Unterricht Gelegenheiten für die Produktion übersatzmäßiger Diskurseinheiten erhalten“. Prediger et al. (2016: 286) konstatieren, dass für den Grundschulunterricht vor allem die Praktiken des Erzählens und Berichtens relevant seien, während ab der fünften Klasse Erklären und Argumentieren zu den wichtigsten Gattungen gehören (vgl. Morek, Heller \& Quasthoff 2017), wie auch jeweils Morek (2016) und Heller et al. (2017) anhand von Gattungsrepertoires für Fachunterricht in fünften Klassen zeigen. Für Daten aus der Grundschule hat Heller (2012) ein Gattungsrepertoire für elf ausgewählte Kinder aus vier verschiedenen Klassen ihres Korpus aufgestellt. Lokale Aktivitäten wie „Begrüßen, Aufgabenstellen und Ergebnisse präsentieren sowie die Hausaufgabenansage“ (Heller 2012: 185) kommen in allen untersuchten Klassen vor. Sie dienen der Herstellung der in- 
stitutionellen Strukturen, stellen jedoch „keine gesprächsstrukturellen Anforderungen im Sinne der Bearbeitung globaler Zugzwänge oder ein- oder ausleitender gesprächsstruktureller Jobs“ (Heller 2012: 185). Globale Anforderungen wie Begründen und Argumentieren, persönliche Erlebniserzählung, Erklären und Beschreiben werden dagegen in den Klassen in unterschiedlichem Ausmaß gefordert ${ }^{3}$. Die Befunde zeigen, dass Lehrpersonen höchst unterschiedlich den Unterricht gestalten und in Folge dessen unterschiedliche diskursive Anforderungen im Sinne globaler Zugzwänge stellen.

Hausendorf \& Quasthoff (2005: 310-314) zufolge besteht im Grundschulunterricht eher wenig Platz für schülerseitige, eigenständig produzierte diskursive Praktiken. „Die Kinder haben nur die Möglichkeit, Ein-Satz-, oft auch nur EinWort-Äußerungen in die dafür vorgesehenen Slots zu stecken“ (Hausendorf \& Quasthoff 2005: 313). Selbst wenn diskursive Anforderungen gestellt werden, werden sie nicht selten zergliedert, indem die Lehrperson zentrale Jobs übernimmt oder aber diese auf mehrere Schülerinnen und Schüler aufteilt, wie auch Morek (2012) in Bezug auf Erklärungen in der Grundschule feststellt. Vielfach werden also globale Zugzwänge auf lokale heruntergebrochen, was den Schülerinnen und Schülern eben dadurch die Chance auf den Erwerb selbstständig produzierter Diskurseinheiten bzw. Äußerungspakete erschwert. Ein Grund für dieses Vorgehen könnte sein, dass Lehrkräfte versuchen, möglichst viele Schülerinnen und Schüler am Diskurs zu beteiligen (Heller 2012; Morek 2012). So wird die Aufmerksamkeit aller Schülerinnen und Schüler zu sichern versucht, um Unruhe und Nebenkommunikationen zu vermeiden (vgl. auch Becker-Mrotzek \& Quasthoff 1998).

Trotz der Fülle an Studien weisen die Befunde darauf hin, dass diskursive Anforderungen vor allem im Grundschulunterricht noch nicht hinreichend untersucht sind. Es lassen sich zwar verschiedene diskursive Praktiken im Unterricht der Grundschule finden: Generell ist Erzählen als alltagsnahe Praxis in der Grundschule, vor allem in den ersten Schuljahren, vielfach zu erwarten (Ohlhus 2014). Auch Erklären (Morek 2012) und Argumentieren (Heller 2012) sowie Beschreiben und Berichten kommen im Unterrichtsgespräch vor. Allerdings ist über die Etablierung solcher diskursiven Anforderungen bisher noch wenig bekannt. Die in Abschnitt 4 vorgestellten Fallbeispiele beleuchten dieses Forschungsdesiderat.

3 In einer Klasse werden hauptsächlich im Erzählkreis globale Anforderungen gestellt, Argumentieren tritt dort ausschließlich als Nebenaktivität auf. In einer weiteren Klasse werden diskursive Anforderungen im Klassengespräch sehr selten gestellt, dann vornehmlich solche zur Begründung und Erklärung. Die dritte Klasse zeigt Diskursaktivitäten in Zusammenhang mit schriftlichen Texten, eingefordert wurden vor allem Begründungen und vereinzelt Begriffserklärungen. In der letzten Klasse gab es mehr Gelegenheiten für lehrerseitig eingeforderte Argumentationen (Heller 2012: 185-186). 


\section{Datengrundlage und analytisches Vorgehen}

Für die Rekonstruktion diskursiver Anforderungen in der Grundschule wird auf ein bestehendes Korpus zurückgegriffen, das 18 Unterrichtsstunden zweier Grundschulklassen im Ruhrgebiet im Verlauf der ersten beiden Schuljahre umfasst. Der Unterricht wurde ungefähr einmal pro Monat ab dem Ende des ersten Schulhalbjahres videographiert. Das Korpus wurde im Rahmen des DFG-Forschungsprojekts Diskursstile als sprachliche Sozialisation (DASS) ${ }^{4}$ unter Leitung von Prof. Dr. Uta Quasthoff von Dezember 2000 bis Dezember 2001 erhoben. Die Aufnahmen zeigen Deutsch- und Sachunterricht bei der jeweiligen Klassenlehrerin. Es wurden den Lehrerinnen keinerlei Vorgaben gemacht, so dass die Aufnahmen weitestgehend natürlichen Unterricht zeigen.

Für die Analysen wurden die Videosequenzen nach den Konventionen von GAT2 (Selting et al. 2009) transkribiert, die im Anhang dieses Bandes einzusehen sind. Die Analyse folgt den Prinzipien der Konversationsanalyse und zeigt ein strikt sequenziell-rekonstruktives Vorgehen (Heller \& Morek 2016).

Prinzipiell gibt es zwei Möglichkeiten, wie Äußerungspakete durch die Lehrkräfte im Unterricht eingefordert werden: Der ,klassische Fall‘ der Etablierung diskursiver Anforderungen ist, so könnte man zunächst annehmen, dass diese im Zugzwang der Lehrperson explizit etabliert werden, wie z. B. in folgender fiktiver Äußerung der Lehrperson: „Wer erklärt mir denn einmal, wie Kartoffeln angebaut werden?“ Ein solcher Zugzwang expliziert sehr deutlich nicht nur den geforderten Inhalt der Äußerung, sondern fordert auch eine spezifische diskursive Gattung ein, nämlich die des Erklärens. Eine einzelne, nicht übersatzmäßige Äußerung wird hier kaum den Ansprüchen der Lehrperson genügen. Diese explizite Setzung von diskursiven Anforderungen unmittelbar im Zugzwang des first turn erscheint logisch, aber es ist nicht der Regelfall (vgl. Heller \& Morek 2015 für die Sekundarstufe I). Vielfach werden diskursive Anforderungen auch erst nachträglich sichtbar. Das bedeutet, dass erst durch die Rückmeldung der Lehrperson auf einen Schülerbeitrag deutlich wird, welche globalen oder lokalen Anforderungen gelten. Insofern werden in den nachfolgenden Analysen stets größere Sequenzen betrachtet, die lehrerseitige Zugzwänge, Schülerbeiträge sowie lehrerseitige Rückmeldungen umfassen.

Eine zweite Möglichkeit ist die explizite Einforderung einer Elaborierung eines Schülerbeitrags oder einer Reparatur. In diesen Fällen gelten die diskursiven

$4 \mathrm{http}$ //home.edo.tu-dortmund.de/ quasthoff/dass/indexdass.html, zuletzt abgerufen am 25.10.2016. Der Abschlussbericht ist online verfügbar unter http://home.edo.tu-dortmund.de/ quasthoff/dass/DASS\%20Abschlussbericht_neu1.pdf, zuletzt abgerufen am 25.10.2016. 
Anforderungen erst für die Überarbeitung des Beitrags, indem z. B. eingefordert wird, dass eine Schülerin oder ein Schüler seine Äußerung erklärt, begründet etc. Die Etablierung diskursiver Erwartungen ist dabei sehr explizit. Diese Fälle sollen hier nicht im Fokus stehen. Es geht im vorliegenden Artikel vielmehr darum, die impliziten oder auch nur scheinbaren diskursiven Anforderungen zu rekonstruieren, die von der Lehrperson etabliert werden. Es wird analysiert, ob und wenn ja, wie ein Zugzwang in eine bestimmte Gattung steuert und welche metadiskursiven Hinweise dabei gegeben werden. Dabei wird auch die Rolle von Sprechhandlungsverben wie z. B. ,erzählen` thematisiert.

\section{Diskursive Anforderungen im Grundschulunterricht}

Diskursive Anforderungen werden also einerseits im Zugzwang der Lehrperson und andererseits in den Rückmeldungen zu Schülerbeiträgen offenbar. Die nachfolgenden Analysen versuchen diese Möglichkeiten zu beleuchten. Dabei werden Explizitheit und Implizitheit der Anforderungsetablierungen ebenso thematisiert wie die Rolle von lehrerseitig gesetzten Zugzwängen und Rückmeldungen auf die von den Schülerinnen und Schülern angebotenen Beiträge. Analog zur Vorstellung vorkommender diskursiver Gattungen im Grundschulunterricht (Abschnitt 2.2) stellt sich zudem die Frage, ob es sich bei den Anforderungen tatsächlich um explizit globalstrukturelle handelt. Die sequenzielle Rekonstruktion bietet so einerseits einen Einblick in die Unterrichtswirklichkeit und die Produktion von Äußerungspakten im Unterricht, andererseits kann durch die Rekonstruktion gezeigt werden, wie komplex das Zusammenspiel zwischen den Äußerungen von Lehrperson und Schülerin(nen) und Schüler(n) ist.

\section{1 (Implizite) Etablierung diskursiver Anforderungen im Unterrichtsgespräch durch die Lehrperson}

In diesem Abschnitt geht es zunächst um die grundsätzliche Etablierung diskursiver Anforderungen im Unterrichtsdiskurs, die vielfach sehr implizit und mitunter sogar unbestimmt verläuft.

Im Fokus des ersten Fallbeispiels steht das Sprechhandlungsverb erzählen. Es handelt sich um eine Stunde, in der die Lehrerin Frau Schmidt den Körperbau und die Haltung eines Kaninchens thematisiert. 
Beispiel 1: A-F-1, Was braucht ein Kaninchen

200 L SO.

$201 \quad$ und ähm die/ bei der pauLIna hab ich schon geSEHen?

202 und (.) bei der ANna?

203 bei der kaTRI:n?

204 (.) hab ich schon auf de:m_äh (.) ARbeitsblatt gesEhen?

205 dass sie auch ANgekreuzt haben,

206 was das kanInchen alles !BRAUCHT! ,

$207 \mathrm{X}$ ((unv.))

208 Y ja: . =hab ich doch $\mathrm{AU}: \mathrm{CH}$.

209 L ((TOM)). willst DU sonst vielleicht sagen.

210 Dir? nein ICH.

211 L DIRK.

261 L WER möchte mal als NÄCHstes was dazu erzählen.

$262 \quad$ ((. . . ca. 10s ))

263 Den ALso.

264 es BRAUCHT ein klei/ eine TRINKflasche?

265 L <<bestätigend>hm_hm?>

266 Den und zwEI FUTternäpfe?

267 L <<bestätigend> hm_hm?>

268 Den? einen WEIßen,

269 XX ((Unruhe))

270 Den ((unv.))

271 ich WEIß nicht-=

272 =wie das HEIßt,

273 So_n FUTternapf; =

$274=$ glaub_ich.

275 so_ne ART,

276 da wo das (.) HEU: : rEi:nko:mmt.

277 L <<bestätigend>hm_hm?>

278 XX ((Kinder reden durcheinander))

279 Den und die brauchen kaNINchenfutter;

280 L <<bestätigend>hm_hm?>

281 XX ((Unruhe, Kinder machen durcheinander weitere Vorschläge)

$282 \mathrm{~L}$ okAY.

283 DANkeschÖn-

284 ((leitet zur nächsten Unterrichtsphase über)) 
In dem gezeigten Ausschnitt sollen die Schülerinnen und Schüler ,erzählen`, was man für die Haltung eines Kaninchens benötigt (Z. 261). Die Anforderungen an den Schülerbeitrag erscheinen zunächst als Einforderung einer Erzählung, also einer diskursiven Praktik, wenn man vom Sprechhandlungsverb ausgeht. Dennis erhält den floor und liefert auf den Zugzwang der Lehrerin eine Aufzählung, ein reines Benennen verschiedener Gegenstände und Materialien. Sein Beitrag weist keine Merkmale einer Erzählung auf. Frau Schmidt unterstützt Dennis in der Produktion des Beitrags durch bestätigende Hörerrückmeldungen (Z. 265, 277, 280), die als continuer (Schegloff 1982) dienen und ihm schon während seiner Äußerung Angemessenheit signalisieren. Sie markiert an keiner Stelle Divergenz, auch nicht in Bezug auf die Nicht-Erfüllung der Realisierung einer Erzählung. Es reicht also offenbar eine Aufzählung; ein narratives Äußerungspaket wird nicht eingefordert, auch wenn im Zugzwang (Z. 261) das Schlüsselwort erzählen auftaucht, dessen Bedeutung eine andere sprachliche Realisierung des Beitrags erwarten ließe. Die akzeptierenden Rückmeldungen lassen jedoch darauf schließen, dass die Aufzählung, also das reine Benennen der Gegenstände, aus Sicht der Lehrperson angemessen ist. Ebenso angemessen ist es offenbar, eine $\mathrm{Be}$ schreibung eines Gegenstands zu liefern, wenn der Begriff selbst nicht bekannt ist (Z. 270-276). Interessant ist dabei, dass die Lehrperson nicht das entsprechende Wort ,Heuraufe“ einfordert oder selbst liefert, obwohl Dennis sogar anzeigt, dass er das Wort sucht, was ein Eingreifen der Lehrperson im Sinne einer vom Schüler eingeforderten Hilfestellung problemlos ermöglichen würde. Sie geht auf die von Dennis produzierte Beschreibung nicht weiter ein und markiert durch ein bestätigendes $h m \_h m$ ? (formale) Angemessenheit. ${ }^{5}$ Durch den Umgang mit Dennis' Beiträgen wird also im Nachhinein deutlich, dass der Zugzwang keine Erzählung etabliert. Nur das erwähnte Schlüsselwort deutet auf diese diskursive Praktik hin. Im Kontext betrachtet macht eine Erzählung auch wenig Sinn. WER möchte mal als NÄCHstes was dazu erzählen. (Z. 261) bedeutet in diesem Kontext also etwas sagen, beitragen, den Zugzwang bedienen. Das kann lokal durch eine Aufzählung getan werden oder aber durch diskursive Praktiken wie Beschreiben. Die Anforderungen sind also relativ offen.

5 Dieses Vorgehen ist typisch für Frau Schmidt. Die Analysen der Lehrer-Schüler-Interaktion weisen insgesamt eine geringe inhaltliche Steuerung auf, was sich vor allem darin zeigt, dass sie vielfach auf formaler Ebene Passung markiert, aber wenig auf inhaltliche Aspekte eingeht (siehe die Analysen zur Herstellung von Angemessenheit in Buttlar 2017b). Morek (2012), die das gleiche Korpus unter dem Gesichtspunkt von Erklärungen im Unterricht untersucht hat, konnte eine formorientierte Erklärpraxis in Bezug auf Frau Schmidts Umgang mit Erklärungen aufzeigen. Diese Erkenntnis lässt sich auf ihr professionelles Verhalten im Umgang mit Schülerinnen und Schülern insgesamt ausweiten. 
Das wird deutlich, wenn man den Vorlauf zu dieser Sequenz betrachtet, in dem die Lehrerin Frau Schmidt das Thema etabliert (Z. 200-211). Unter Einbezug vorheriger Äußerungen wird deutlich, dass die Kinder ein Aufgabenblatt vorliegen haben, auf dem sie auf einer Liste ankreuzen sollten, was ein Kaninchen alles braucht. Daran anschließend fordert Frau Schmidt die Schülerinnen und Schüler auf, etwas dazu zu sagen (Z. 209). Der erste Zugzwang ist inhaltlich unspezifisch und lässt auch sprachlich völlig offen, was in diskursiver Hinsicht erwartet ist. Die Aufforderung willst DU sonst vielleicht sagen. (Z. 209) bezieht sich aber auf das Aufgabenblatt, so dass das Benennen der Dinge, was das kanInchen alles !BRAUCHT!, (Z. 206), als angemessene Bedienung des Zugzwangs gelten kann, da hier offensichtlich die inhaltlichen Aspekte im Vordergrund stehen.

Unter diesen Gesichtspunkten bedeutet die Aufgabenstellung aus dem Beispiel 1 ,etwas dazu erzählen' (Z. 261) also, etwas wiederzugeben bzw. aufzuzählen. In dem Zugzwang von Frau Schmidt wird - abgesehen vom Schlüsselwort erzählen - an keiner Stelle eine globale, d. h. diskursive Anforderung explizit gemacht (zur Verwendung derartiger ,Schlüsselwörter' vgl. Morek 2016, Kotthoff 2009). Um zu erkennen, welche (diskursiven) Anforderungen von der Lehrperson etabliert werden, reicht es also nicht allein aus, das verwendete Sprechhandlungsverb der Lehrperson zu beachten. Vielmehr müssen Schülerinnen und Schüler auch den den Zugzwang umgebenden Kontext erkennen und diesen ,in seinem sowohl thematischen als auch diskursiven Verlauf mitprozessier[en] und bei der Umsetzung der betreffenden Diskurspraktik(en) berücksichtig[en]“ (Morek 2016: 111). Darüber hinaus können, wie im Beispiel 1 gezeigt, die Rückmeldungen der Lehrperson auf die Schüleräußerung Hinweise auf diskursive Anforderungen geben, wobei ein sehr implizites Vorgehen eindeutige Hinweise oftmals verhindert.

Drei Dinge wurden an diesem ersten Ausschnitt aus einer Grundschulstunde deutlich:

1. In Zugzwängen verwendete ,diskursive Schlüsselwörter‘ können durchaus andere Anforderungen als die semantisch erwartbaren generieren. Das zeigt sich auch in anderen Sequenzen aus dem vorliegenden DASS-Korpus, vor allem im Zusammenhang mit dem Sprechhandlungsverb erzählen. Prinzipiell sind diskursive Erwartungen in Zugzwängen selten explizit zu finden.

2. Der einen Zugzwang umgebende Kontext ist entscheidend für die Produktion eines angemessenen Beitrags. Schülerinnen und Schüler müssen ihren Beitrag in diesen Kontext einpassen.

3. Zugzwänge können sehr vage in der Etablierung von Anforderungen sein. Ob ein Beitrag einer Schülerin oder eines Schülers angemessen ist und auf inhaltlicher wie auch sprachlich-formaler, gesprächsorganisatorischer oder 
globalstruktureller Ebene akzeptiert wird, wird oft erst deutlich, wenn man die Rückmeldungen der Lehrperson auf die Schülerbeiträge genauer betrachtet. Vielfach werden die Anforderungen auch dort erst deutlich, oft allerdings nur implizit.

Tatsächlich lassen sich im DASS-Korpus nur wenig sequenzeröffnende Zugzwänge finden, in denen diskursive Anforderungen explizit gemacht werden.

In einem anderen Beispiel aus der Klasse A erscheint die Etablierung diskursiver Anforderungen im sequenzeröffnenden Zugzwang wesentlich deutlicher zu sein. Im Fokus stehen dort formale Aspekte, d. h. das Beitragen an sich wird von der Lehrerin in der Unterrichtsstunde relevant gesetzt (vgl. Morek 2012). Frau Schmidt liest eine Geschichte von einem Außerirdischen vor, der in das Weltall fliegt und dort in Richtung eines blauen Planeten abstürzt. Die Schülerinnen und Schüler sollen anschließend die Geschichte weitererzählen bzw. SELBST was sagen. (Z. 062).

Beispiel 2: A-F-2, Luno und der blaue Planet (I)

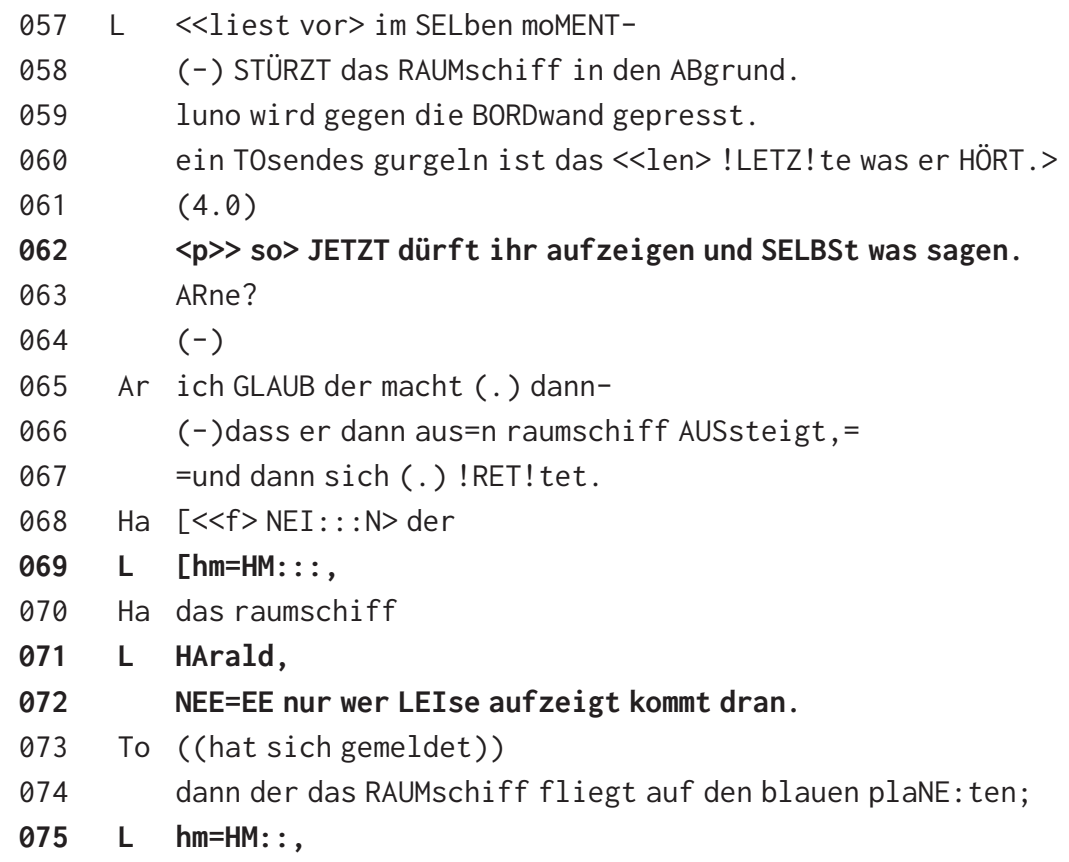


Der Zugzwang ist wie in den vorigen Beispielen hinsichtlich der kommunikativen Erwartung unterbestimmt. Im Anschluss an den vorgelesenen Text wird lediglich die Aufforderung expliziert, sich am Unterricht $\mathrm{zu}$ beteiligen (gesprächsorganisatorische Ebene). ,Etwas sagen' muss nicht zwangsläufig ,die Geschichte weitererzählen` bedeuten (siehe oben Punkt 1). Im Kontext einer vorgelesenen Geschichte, die an einer spannenden Stelle unterbrochen wird, und einer anschließenden Beitragsaufforderung an die Schülerinnen und Schüler ist dieses Weitererzählen aber durchaus erwartbar (siehe oben Punkt 2). Die Sequenz zeigt somit sehr deutlich, dass der oben angesprochene Punkt 3, die Relevanz der lehrerseitigen Rückmeldungen, von elementarer Bedeutung ist: Die ersten Schülerinnen und Schüler, die sich melden, schließen inhaltlich zwar an den Kontext der fiktiven Geschichte an. Allerdings nennen sie nur eine für sie denkbare Möglichkeit, wie es weitergeht. Eine Erzählung im Sinne einer übersatzmäßigen Einheit liefern sie nicht. Dennoch werden die Äußerungen gewürdigt durch ein bestätigendes $h m=H M$, (Ehlich 1986). Sie werden von der Lehrperson also als angemessen markiert, obwohl sie nur lokalen Charakter haben. Damit wird offensichtlich, dass die Lehrerin eine globale Bedienung nicht zwingend einfordert, obwohl eine Bedienung dieser Art im Sinne eines Weitererzählens durch den umgebenden Kontext durchaus naheliegt.

Dieses Rückmeldemuster zieht sich durch die Unterrichtsstunde. Auch im weiteren Verlauf werden die Schülerinnen und Schüler nicht bei der Produktion von Äußerungspaketen unterstützt. Frau Schmidt beschränkt ihre Aktivitäten darauf, die kommunikative Ordnung durch Disziplinierungen aufrechtzuerhalten (wie in Z. 071-072), die Schülerbeiträge selbst werden lediglich minimalratifiziert (Buttlar 2017b), d. h. formal als angemessen markiert (wie in Z. $069+075$ ).

Durch die mangelnde inhaltliche Steuerung verlagert sich das Thema im weiteren Verlauf auf Sachwissen zum Weltall und die Beiträge haben zu dem ursprünglichen Ausgangspunkt nur noch wenig Bezug. Die Einzelbeiträge lassen sich nicht zu einer kohärenten Weitererzählung zusammenfassen, auch sonst besteht wenig Kohärenz in Bezug auf einen Unterrichtsgegenstand, der im Klassengespräch behandelt werden soll. Frau Schmidt etabliert lediglich die formale Erwartung, dass die Schülerinnen und Schüler sich beteiligen sollen. Diese Erwartung ist allerdings nicht sehr spezifisch: Inhaltliche und globalstrukturelle Anforderungen werden dabei nur vereinzelt im Rahmen von lehrerseitigen Nachfragen zu einem Schülerbeitrag etabliert. So wird auch im Rahmen dieser Unterrichtsstunde, wie in Beispiel 1, die Formulierung ,etwas erzählen' wenig spezifisch verwendet, d.h. gleichgesetzt mit ,etwas 
sagen`. Frau Schmidt stellt diesen Zusammenhang im Rahmen einer Disziplinierung selbst her:

Beispiel 3: Was erzählen

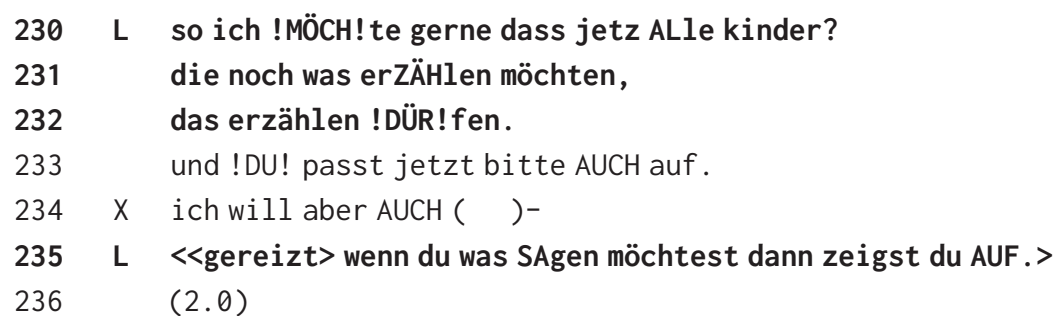

In der Disziplinierung wird deutlich, dass in dieser Unterrichtsstunde die Einhaltung der kommunikativen Ordnung und ein Beitragen im Rahmen dieser Ordnung als angemessene Partizipation von den Schülerinnen und Schülern gefordert wird, es also vor allem um formale Aspekte geht. Das Schlüsselwort ,erzählen' wird dabei sehr frei verwendet und lässt keinen genauen Rückschluss auf geforderte Diskursaktivitäten zu. Der kommunikative Erfolg der Schülerinnen und Schüler wird daher erst in den Rückmeldungen der Lehrperson sichtbar, wobei sie prinzipiell alle Beiträge akzeptiert, die den formalen Anforderungen genügen. Dazu zählt, dass die Schülerinnen und Schüler durch Melden anzeigen sollen, dass sie einen turn übernehmen möchten, und diesen erst nach Aufrufen durch die Lehrperson äußern. Werden diese Anforderungen nicht erfüllt, folgt eine Disziplinierung oder eine nachträgliche Autorisierung zur Übernahme des turns. Inhaltlich und globalstrukturell stellt die Lehrperson keine expliziten Anforderungen, auf diesen Ebenen scheint das Prinzip ,anything goes‘ zu gelten.

Es zeigt sich in den bisherigen Beispielen neben einer großen Implizitheit in der Etablierung von (diskursiven) Anforderungen, dass vielfach erst die Rückmeldungen offenbaren, was die Lehrperson als angemessen betrachtet, $d$. h. welche Anforderungen globaler oder lokaler Art gestellt werden. Wenn man also diskursive Anforderungen im (Grundschul-) Unterricht betrachten will, darf man sich nicht nur auf die Zugzwänge der Lehrperson konzentrieren. Die Rekonstruktion der Anforderungen, die eine Lehrperson an die Schülerinnen und Schüler stellt, muss vor allem die Reaktionen der Lehrperson auf Schüleräußerungen miteinbeziehen, wie es in den obigen Analysen vollzogen wurde. Das wird vor allem im nächsten Abschnitt deutlich. Während in den bisher vorgestellten Beispielen globale Beiträge der Schülerinnen und Schüler prinzipiell möglich, aber nicht zwingend erwartet sind, wird im nächsten Abschnitt ein Beispiel vorgestellt, in dem tatsächlich globale Anforderungen etabliert werden. 


\subsection{Lehrerseitige Einforderung globaler Aktivität}

Im vorliegenden Abschnitt soll ein Beispiel vorgestellt werden, in welchem die Lehrperson eine globale Bedienung des Zugzwangs implizit einfordert. Das Beispiel stammt aus einer Unterrichtsstunde zum Thema ,Leben in der Arktis'. Ausgangspunkt ist eine Gruppenarbeit, in der die Schülerinnen und Schüler Bilder zur Arktis auf spezifische Merkmale dieses Lebensraums hin beschreiben sollten.

Beispiel 4: B-F-3, Was habt ihr herausgefunden (I)

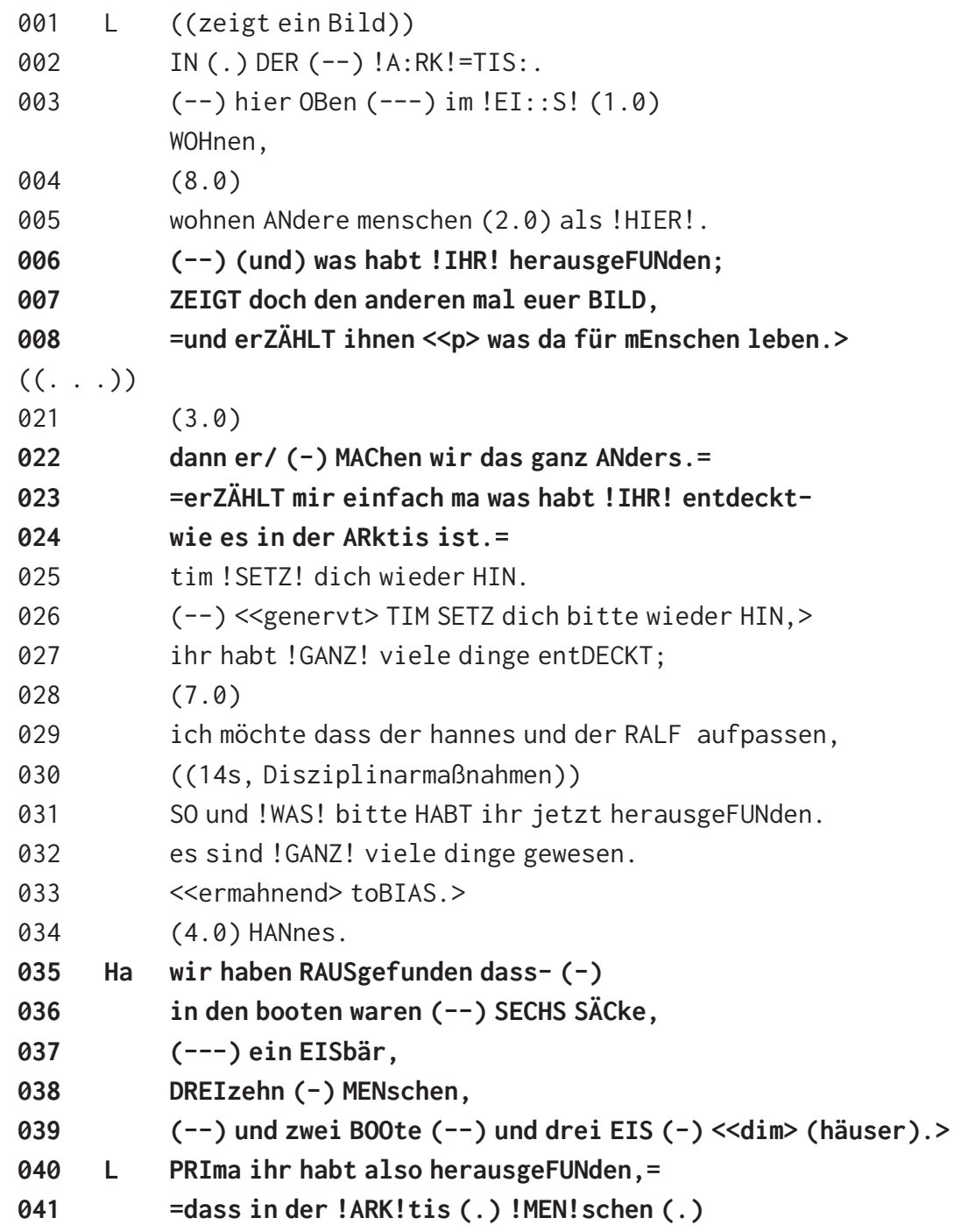


042

mit !B00! ten unterwegs sind.

$043 \quad(-)$ und die irgendwas in den !B00! ten (-) WEGbringen,

$044(--)$ und die gAnz NA: : H bei EISbären leben.

$045 \quad(--)$ was habt ! IHR! entdeckt toBIas.

Der die Präsentation der Gruppenarbeit anleitende Zugzwang enthält wie in den vorherigen Beispielen das Sprachhandlungsverb erzählen, wobei auch hier die Art der Anforderung zunächst nicht weiter spezifiziert wird. So liefert der in Z. 034 aufgerufene Hannes eine detaillierte Auflistung der Dinge auf dem Bild. Dieses reine Benennen stellt keine globale Bedienung dar. Dass die Lehrperson eine solche Bedienungsart aber relevant macht, lässt sich nun abermals implizit in der lehrerseitigen Rückmeldung erkennen: In der Bewertung der Schüleräußerung wird zwar zunächst ein Lob geäußert (Z. 040), aber anschließend greift die Lehrerin den Schülerbeitrag im Rahmen eines Umformulierens (Bührig 1996) auf, d.h. sie formuliert den Schülerbeitrag zu einem kohärenten Äußerungspaket um, einer Beschreibung, bei der zudem die detaillierten Mengenangaben, die Hannes macht, getilgt und damit in der Relevanz zurückgestuft werden. Das Umformulieren zeigt inhaltliche Akzeptanz an, überarbeitet aber gleichzeitig die globalstrukturelle Ebene (Buttlar 2017b). Damit zeigt sie, welche sprachliche Form angemessen ist, und gibt so ein Muster für weitere Beiträge (vgl. Hausendorf \& Quasthoff 2005). Die diskursiven Anforderungen sind damit sehr implizit, zumal das vorangehende Lob (Z. 040) die lehrerseitige Überarbeitung verschleiert.

Für die Schülerinnen und Schüler stellt sich nun die besondere Herausforderung, in der Rückmeldung der Lehrperson ein Muster für weitere, ähnliche Beiträge zu sehen, die eingefordert werden (siehe der Aufruf von Tobias, Z. 045). Tatsächlich zeigt bereits der nächste aufgerufene Schülerbeitrag eine komplexere Struktur. Zwar beginnt Tobias seinen Beitrag in der gleichen Form wie Hannes, geht dann allerdings über zu einer Beschreibung:

Beispiel 5: B-F-3, Was habt ihr herausgefunden (II)

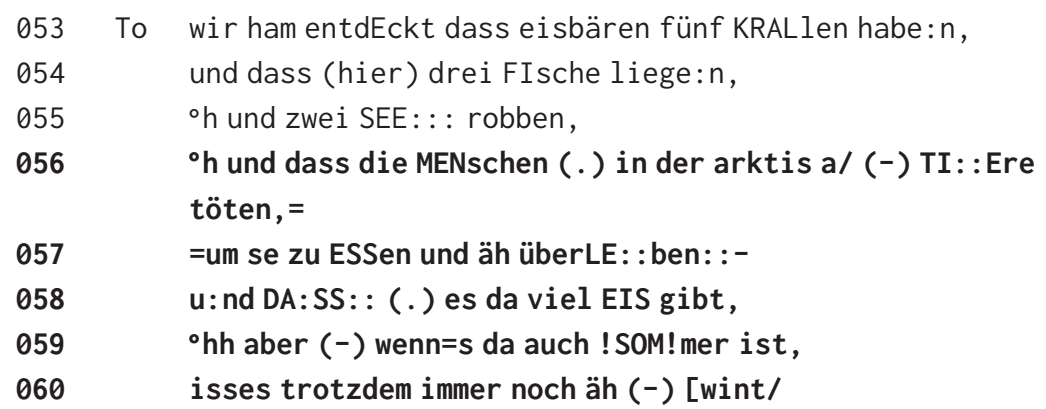


$061 x$

[EIS

062 To ä: hm isses da tr0tzdem noch ähm EI:S.

063 L GANZ prima.

064 auch im <<Zählgeste „1“> ! SOM!mer> ist da EI : :S?

$065(--)$ die menschen gehen zur <<Zählgeste „2“> JA: :GD?>

$066 \quad(--)$ ihr habt herausgefunden WAS gejagt wird?

$067 \quad(1.0)<<t$, all $>$ sachst=du=s nochma toBIas?>

068 To ä:hm SEErobben=fische: :,

069 EISbären,

$070 \quad u:$ :nd ä: :h (--) (die jagen) FISCH.

$071 \mathrm{~L}$ joa das is ja auch schon ne !MEN!ge.

072 To $H M=[H M$,

073 L [der eisbär wird ! AU:CH! gejagt;=

$074=$ =zum !ES! sen?

075

aber nIicht nur zum !ES! sen; =

Auch diese Äußerung wird von Frau Mustermann gelobt (Z. 063) und ebenfalls umformuliert, wobei hier der Aspekt der Kohärenz im Fokus steht. Sie greift aber explizit die Jagdbeschreibung von Tobias auf und bindet ihn in das weitere Unterrichtsgespräch ein, das sich zunächst genauer mit der Jagd und dem Zweck dieser Tätigkeit beschäftigt. In diesem Sinne hat Tobias mit der Platzierung einer (anfänglichen) globalen Bedienung kommunikativen Erfolg: Die von ihm beschriebene Jagd der Menschen in der Arktis wird zum Thema für das Unterrichtsgespräch gemacht; im weiteren Verlauf der Sequenz (aus Platzgründen nicht abgebildet) wird interaktiv im Klassengespräch Wissen über die Jagd und die Nützlichkeit des Eisbären aufgebaut. So dokumentiert sich in Tobias' Strategiewechsel in Z. 056 sein Verständnis, dass ein reines Benennen und Aufzählen zur angemessenen Bedienung des Zugzwangs nicht ausreicht, sondern die Lehrperson implizit eine globale Bedienung einfordert.

Diese Unterrichtssequenz zeigt wie die vorherigen Beispiele auch, dass Zugzwänge der Lehrperson oftmals hinsichtlich ihrer kommunikativen Erwartungen unbestimmt und zudem implizit gesetzt sind. Vor allem aber wird deutlich, dass die diskursiven Anforderungen besonders in der Rückmeldung der Lehrperson sichtbar werden. Allerdings sind sie auch an dieser sequenziellen Position hochgradig implizit und verschleiert durch ein Lob. Es erfordert somit nicht nur kontinuierliche Aufmerksamkeit seitens der Schülerinnen und Schüler, sondern auch erhebliche inferentielle Anstrengungen, um kommunikative Erwartungen zu erkennen. Obwohl die Lehrperson hier also tatsächlich diskursive Anforderungen stellt und eine lokale Bedienung des Zugzwangs nicht akzeptiert, bleiben Globalität und Gattungsorientierung implizit. 


\section{Fazit und Ausblick}

Der Artikel beschäftigte sich mit diskursiven Anforderungen im mündlichen Unterrichtsdiskurs, die in der Grundschule von der Lehrperson gestellt werden. Es wurde anhand exemplarischer Beispiele aus dem Grundschulunterricht beleuchtet, auf welche Weise diskursive Anforderungen durch die Lehrperson gestellt werden. Dabei standen solche Sequenzen im Fokus, die nur implizit diese Anforderungen initiieren und zuweilen anhand des Sprechhandlungsverbs erzählen auch irreführende Anforderungen stellen.

Die Befunde der Analysen lassen sich wie folgt zusammenfassen: Es bestehen im Klassengespräch nicht immer klare kommunikative Erwartungen bezüglich globalstruktureller und auch inhaltlicher Aspekte. Die einzige in allen Beispielen zu rekonstruierende Anforderung besteht darin, dass die Schülerinnen und Schüler sich beteiligen sollen, was eine klare Anforderung auf gesprächsorganisatorischer Ebene darstellt. Damit ist aber lediglich die Grundeigenschaft von Zugzwängen erfüllt: Zugzwänge setzen ihre Bedienung durch die weiteren Gesprächsbeteiligten hochgradig relevant oder erfordern vor allem im lehrergeleiteten Klassengespräch bei Nichtbedienung einen account (vgl. Heller 2012: 211) oder eine Reformulierung (Bührig 1996).

Darüber hinaus konnte gezeigt werden, dass gerade Sprechhandlungsverben wie das hier verwendete erzählen keinen Aufschluss über mit dem Zugzwang etablierte kommunikative Erwartungen geben. In den gezeigten Sequenzen handelt es sich selten um einen ,echten' globalen Zugzwang; die Bedienung des Zugzwangs durch die Schülerinnen und Schüler kann sowohl global als auch lokal erfolgen. Insofern sind der Zugzwang und die damit verbundenen globalen Anforderungen nur sehr unbestimmt formuliert.

Lediglich im letzten Beispiel setzt die Lehrperson eine globale Bedienung des Zugzwangs relevant. Sie fordert zwar den Schüler nicht zur Reparatur auf, sondern signalisiert Angemessenheit, ausgedrückt durch ein Lob. Die anschließende Umformulierung des Beitrags transformiert aber die vom Schüler geäußerten Informationen in eine globale Einheit. Auf diese Weise zeigt die Lehrperson, welche Struktur sie von dem Beitrag erwartet, und fordert so äußerst implizit die Umsetzung einer diskursiven Praktik ein. Sie setzt gleichzeitig die von ihr nachträglich etablierte Gattung Beschreiben mit erkennbarer kommunikativer Funktion relevant: Diese besteht im Aufbau von Wissen, im Beispiel um Wissen über das Leben in der Arktis. Eben diese Funktion unterscheidet Beispiel (4) von den Beispielen (1) - (3): Ein Äußerungspaket ist zur Generierung komplexen fachlichen Wissens notwendig.

Die vorgelegten Analysen zeigen somit, dass Zugzwänge erstens oftmals unbestimmt sind und für die Schülerinnen und Schüler keine erkennbare Funktion 
besitzen; zweitens können sie Sprechhandlungsverben wie erzählen beinhalten, die jedoch keinen Hinweis auf die kommunikative Erwartung geben; drittens werden auch im evaluierenden turn Erwartungen häufig nur implizit vermittelt und setzen erhebliche inferentielle Leistungen seitens der Schülerinnen und Schüler voraus.

Insgesamt lässt sich festhalten, dass die Unbestimmtheit und Implizitheit diskursiver Anforderungen eine Hürde für eine erfolgreiche Partizipation am Unterrichtsgeschehen darstellen kann (Buttlar 2017a). Hier gilt es in sprachdidaktischer Hinsicht, Lehrkräfte in Bezug auf die Funktionen sowie die Einbettung diskursiver Praktiken im Unterrichtsgespräch zu sensibilisieren, um so bessere Partizipationsbedingungen für Schülerinnen und Schüler zu schaffen.

\section{Literatur}

Becker, Tabea \& Petra Wieler (Hrsg.) (2013): Erzählforschung und Erzähldidaktik heute: Entwicklungslinien, Konzepte, Perspektiven. Tübingen: Stauffenburg.

Becker-Mrotzek, Michael (2011): Der Erzählkreis als Exempel für die Besonderheiten der Unterrichtskommunikation. In Christoph Bräuer \& Jakob Ossner, (Hrsg.), Kommunikation und Interaktion im Unterricht, 31-45. Duisburg: Universitätsverlag.

Becker-Mrotzek, Michael \& Uta Quasthoff (1998): Zu diesem Heft: Unterrichtsgespräche zwischen Gesprächsforschung, Fachdidaktik und Unterrichtspraxis. Der Deutschunterricht (1), 3-13.

Becker-Mrotzek, Michael \& Rüdiger Vogt (2009): Unterrichtskommunikation: Linguistische Analysemethoden und Forschungsergebnisse. Tübingen: Niemeyer.

Bührig, Kristin (1996): Reformulierende Handlungen: Zur Analyse sprachlicher Adaptierungsprozesse in institutioneller Kommunikation. Tübingen, Hamburg: Narr.

Buttlar, Ann-Christin (2017a): Implizite Normvermittlung durch Konstituierung von Angemessenheit im Unterrichtsdiskurs. In Stefan Hauser \& Martin Luginbühl, (Hrsg.), Gesprächskompetenz in schulischer Interaktion: Normative Ansprüche und kommunikative Praktiken, 38-64. Bern: hep.

Buttlar, Ann-Christin (2017b): Erwartungen von Lehrpersonen und Äußerungen von Schülerinnen und Schülern: Sequenzielle Analysen zur Ko-Konstruktion von Angemessenheit im Unterrichtsdiskurs. Technische Universität Dortmund Dissertation.

Deppermann, Arnulf, Helmut Feilke \& Angelika Linke (2016): Sprachliche und kommunikative Praktiken: Eine Annäherung aus linguistischer Sicht. In Arnulf Deppermann, Helmuth Feilke \& Angelika Linke, (Hrsg.), Sprachliche und kommunikative Praktiken, 1-24. Berlin, Boston: De Gruyter.

Dubs, Rolf (2009): Lehrerverhalten: Ein Beitrag zur Interaktion von Lehrenden und Lernenden im Unterricht. Stuttgart: Steiner.

Ehlich, Konrad (1986): Interjektionen. Tübingen: Niemeyer.

Ehlich, Konrad (2009): Unterrichtskommunikation. In Michael Becker-Mrotzek, (Hrsg.), Mündliche Kommunikation und Gesprächsdidaktik: Teilband Mündlichkeit, 327-348. Baltmannsweiler: Schneider Hohengehren. 
Ehlich, Konrad \& Jochen Rehbein (1986): Muster und Institution: Untersuchungen zur schulischen Kommunikation. Tübingen: Gunter Narr Verlag.

Erath, Kirstin, Anna Vogler, Susanne Prediger, Vivien Heller \& Uta Quasthoff (2014): Interaktive Verfahren der Enkulturation von Lernenden in fachspezifische Praktiken im Mathematik- und Deutschunterricht. In Jürgen Roth \& Judith Ames, (Hrsg.), Beiträge zum Mathematikunterricht 2014: Beiträge zur 48. Jahrestagung der Gesellschaft für Didaktik der Mathematik vom 10. bis 14. März 2014 in Koblenz, 1349-1350. Münster: WTM Verlag für wissenschaftliche Texte und Medien.

Fienemann, Jutta \& Rainer von Kügelgen (2006): Formen mündlicher Kommunikation in Lehr- und Lernprozessen. In Ursula Bredel, Hartmut Günther, Peter Klotz, Jakob Ossner \& Gesa Siebert-Ott, (Hrsg.), Didaktik der deutschen Sprache: Ein Handbuch. 2. Teilband, 133-147. Paderborn: F. Schöningh.

Grundler, Elke (2011): Kompetent argumentieren: Ein gesprächsanalytisch fundiertes Modell. Tübingen: Stauffenburg.

Grundler, Elke \& Rüdiger Vogt (Hrsg.) (2006): Argumentieren in Schule und Hochschule: Interdisziplinäre Studien. Tübingen: Stauffenburg.

Harren, Inga (2009): Schülererklärungen im Unterrichtsgespräch des Biologieunterrichts. In Janet Spreckels, (Hrsg.), Erklären im Kontext: Neue Perspektiven aus der Gesprächs- und Unterrichtsforschung, 81-93. Baltmannsweiler: Schneider Hohengehren.

Hausendorf, Heiko \& Uta Quasthoff (2005): Sprachentwicklung und Interaktion: Eine linguistische Studie zum Erwerb von Diskursfähigkeiten. Radolfzell: Verlag für Gesprächsforschung.

Heller, Vivien (2012): Kommunikative Erfahrungen von Kindern in Familie und Unterricht: Passungen und Divergenzen. Tübingen: Stauffenburg.

Heller, Vivien (2014): Gesprächsanalyse in der sprachwissenschaftlichen und sprachdidaktischen Unterrichtsforschung. In Astrid Neumann \& Isabelle Mahler, (Hrsg.), Empirische Methoden der Deutschdidaktik: Audio- und videografierende Unterrichtsforschung, 122-150. Baltmannsweiler: Schneider Hohengehren.

Heller, Vivien \& Miriam Morek (2015): Unterrichtsgespräche als Erwerbskontext: Kommunikative Gelegenheiten für bildungssprachliche Praktiken erkennen und nutzen. Leseforum.ch (3), 1-23.

Heller, Vivien \& Miriam Morek (2016): Gesprächsanalyse: Mikroanalytische Beschreibung sprachlicher Interaktion in Bildungs- und Lernzusammenhängen. In Jan Boelmann, (Hrsg.), Empirische Erhebungs- und Auswertungsverfahren in der deutschdidaktischen Forschung, 223-246. Baltmannsweiler: Schneider Hohengehren.

Heller, Vivien, Uta Quasthoff, Susanne Prediger \& Anna Vogler (2017): Bildungssprachliche Praktiken aus professioneller Sicht: Wie deuten Lehrende Schülererklärungen und -begründungen? In Bernt Ahrenholz, Britta Hövelbrinks \& Claudia Schmellentin, (Hrsg.), Fachunterricht und Sprache in schulischen Lehr-/Lernprozessen, 139-160. Tübingen: Narr Francke Attempto.

KMK (2004): Bildungsstandards im Fach Deutsch für den Primarbereich (Jahrgangsstufe 4). Beschluss vom 15.10.2004. https://www.kmk.org/fileadmin/Dateien/veroeffentlichun gen_beschluesse/2004/2004_10_15-Bildungsstandards-Deutsch-Primar.pdf. (Letzter Aufruf am 27.06.2020)

Knapp, Werner (2001): Erzähltheorie und Erzählerwerb. Didaktik Deutsch (10), 228-252. 
Kotthoff, Helga (2009): Gesprächsfähigkeiten: Erzählen, Argumentieren, Erklären. In Michael Krelle \& Carmen Spiegel, (Hrsg.), Sprechen und Kommunizieren: Entwicklungsperspektiven, Diagnosemöglichkeiten und Lernszenarien in Deutschunterricht und Deutschdidaktik, 41-63. Baltmannsweiler: Schneider Hohengehren.

Luckmann, Thomas (1986): Grundformen der gesellschaftlichen Vermittlung des Wissens: Kommunikative Gattungen. In Kultur und Gesellschaft: René König, dem Begründer der Sonderhefte, zum 80. Geburtstag gewidmet, 191-211. Opladen: Westdt. Verlag.

Macbeth, Douglas (2004): The relevance of repair for classroom correction. Language in Society 33 (5), 703-736.

McHoul, Alexander (1990): The Organization of Repair in Classroom Talk. Language in Society 19 (3), 349-377.

Mehan, Hugh (1979a): Learning lessons: Social organization in the classroom. Cambridge, Mass: Harvard University Press.

Mehan, Hugh (1979b): „What Time Is It, Denise?“: Asking Known Information Questions in Classroom Discourse. Theory Into Practice 18 (4), 285-294.

Morek, Miriam (2011): Explanative Diskurspraktiken in schulischen und ausserschulischen Interaktionen: Ein Kontextvergleich. Schweizerische Zeitschrift für Bildungswissenschaften 33 (2), 211-230.

Morek, Miriam (2012): Kinder erklären: Interaktionen in Familie und Unterricht im Vergleich. Tübingen: Stauffenburg.

Morek, Miriam (2013): Erzählkreise: Narrativ eingebettete Erklärsequenzen als authentische Gesprächsanlässe im Unterricht. In Tabea Becker \& Petra Wieler, (Hrsg.), Erzählforschung und Erzähldidaktik heute: Entwicklungslinien, Konzepte, Perspektiven, 73-95. Tübingen: Stauffenburg.

Morek, Miriam (2016): Formen mündlicher Darstellung in situ: Zur Komplexität von Diskursanforderungen in Unterrichtsgesprächen. In Ulrike Behrens \& Olaf Gätje, (Hrsg.), Mündliches und schriftliches Handeln im Deutschunterricht: Wie Themen entfaltet werden, 95-131. Frankfurt am Main: Lang.

Morek, Miriam \& Vivien Heller (2012): Bildungssprache - Kommunikative, epistemische, soziale und interaktive Aspekte ihres Gebrauchs. Zeitschrift für angewandte Linguistik 57 (1), 67-101.

Morek, Miriam, Vivien Heller \& Uta Quasthoff (2017): Erklären und Argumentieren: Modellierungen und empirische Befunde zu Strukturen und Varianzen. In Iris Meißner \& Eva Wyss, (Hrsg.), Begründen - Erklären - Argumentieren: Konzepte und Modellierungen in der Angewandten Linguistik, 11-46. Tübingen: Stauffenburg.

Neuland, Eva, Petra Balsliemke \& Anka Baradaranossadat (2009): Schülersprache, Schulsprache, Unterrichtssprache. In Michael Becker-Mrotzek, (Hrsg.), Mündliche Kommunikation und Gesprächsdidaktik: Teilband Mündlichkeit, 392-407. Baltmannsweiler: Schneider Hohengehren.

Ohlhus, Sören (2014): Erzählen als Prozess: Interaktive Organisation und narrative Verfahren in mündlichen Erzählungen von Grundschulkindern. Tübingen: Stauffenburg.

Ohlhus, Sören \& Juliane Stude (2009): Erzählen im Unterricht der Grundschule. In Michael Becker-Mrotzek, (Hrsg.), Mündliche Kommunikation und Gesprächsdidaktik: Teilband Mündlichkeit in der Handbuchreihe Deutschunterricht in Theorie und Praxis, 471-486. Baltmannsweiler: Schneider Hohengehren. 
Prediger, Susanne, Kirstin Erath, Uta Quasthoff, Vivien Heller \& Anna Vogler (2016): Befähigung zur Teilhabe an Unterrichtsdiskursen: Die Rolle von Diskurskompetenz. In Jürgen Menthe, Dietmar Höttecke, Thomas Zabka, Marcus Hammann \& Martin Rothgangel, (Hrsg.), Befähigung zu gesellschaftlicher Teilhabe: Beiträge der fachdidaktischen Forschung, 285-300. Münster: Waxmann.

Quasthoff, Uta (2011): Diskurs- und Textfähigkeiten: Kulturelle Ressourcen ihres Erwerbs. In Ludger Hoffmann, Kerstin Leimbrink \& Uta Quasthoff, (Hrsg.), Die Matrix der menschlichen Entwicklung, 210-251. Berlin: De Gruyter.

Quasthoff, Uta, Vivien Heller, Susanne Prediger \& Kirstin Erath (submitted): Learning in classroom interaction from the perspectives of interactional discourse analysis and subject matter education.

Quasthoff, Uta \& Friederike Kern (2007): Familiale Interaktionsmuster und kindliche Diskursfähigkeit: Mögliche Auswirkungen interaktiver Stile auf diskursive Praktiken und Kompetenzen bei Schulkindern. In Heiko Hausendorf, (Hrsg.), Gespräch als Prozess: Linguistische Aspekte der Zeitlichkeit verbaler Interaktion, 277-305. Tübingen: G. Narr.

Quasthoff, Uta \& Miriam Morek (2015): Diskursive Praktiken von Kindern in außerschulischen und schulischen Kontexten (DisKo): Abschlussbericht für das DFG-geförderte Forschungsprojekt. http://www.disko.tu-dortmund.de/disko/Medienpool/ Abschlussbericht-DisKo.pdf. (Letzter Aufruf am 27.06.2020)

Sacks, Harvey (1995): Lectures on conversation. Volumes I \& II. Edited by Jefferson Oxford: Blackwell.

Schegloff, Emanuel (1982): Discourse as an Interactional Achievement: Some Uses of ,uh huh and other Things that Come between Sentences. In Deborah Tannen, (Hrsg.) Analyzing discourse: Text and talk, 71-93. Washington, D.C: Georgetown University Press.

Selting, Margret, Peter Auer, Dagmar Barth-Weingarten, Jörg Bergmann, Pia Bergmann, Karin Birkner, Elizabeth Couper-Kuhlen, Arnulf Deppermann, Peter Gilles, Susanne Günthner, Martin Hartung, Friederike Kern, Christine Mertzlufft, Christian Meyer, Miriam Morek, Uta Quasthoff, Wilfried Schütte, Anja Stukenbrock \& Susanne Uhmann (2009): Gesprächsanalytisches Transkriptionssystem 2 (GAT 2). Gesprächsforschung - OnlineZeitschrift zur verbalen Interaktion (10), 353-402.

Spiegel, Carmen (1999): Argumentation von Jugendlichen im Deutschunterricht: Zwei Argumentationsformen. Zeitschrift für angewandte Linguistik (30), 17-40.

Spiegel, Carmen (2006): Unterricht als Interaktion: Gesprächsanalytische Studien zum kommunikativen Spannungsfeld zwischen Lehrern, Schülern und Institution. Radolfzell: Verlag für Gesprächsforschung.

Spreckels, Janet, (Hrsg.) (2009): Erklären im Kontext: Neue Perspektiven aus der Gesprächsund Unterrichtsforschung. Baltmannsweiler: Schneider Hohengehren.

Streeck, Jürgen (1979): Sandwich. Good for you.: Zur pragmatischen und konversationellen Analyse von Bewertungen im institutionellen Diskurs der Schule. In Jürgen Dittmann, (Hrsg.), Arbeiten zur Konversationsanalyse, 235-257. Tübingen: Niemeyer.

Vogt, Rüdiger (1998): Lehrer Macht Unterricht: Zur Normalitätskonstruktion im Klassenzimmer. Osnabrücker Beiträge zur Sprachtheorie (OBST) (57), 117-136.

Wald, Benji (1978): Zur Einheitlichkeit und Einleitung von Diskurseinheiten. In Uta Quasthoff, (Hrsg.), Sprachstruktur, Sozialstruktur: Zur linguistischen Theorienbildung, 128-149. Kronberg/Taunus: Scriptor-Verlag. 